National Bureau of Standarc

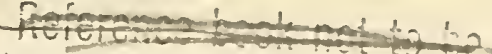

Library, E-0I Admin. Bldg. takeit flefin the ithtary.

\title{
The Brake Pedal Force Capability of Adult Females
}

U.S. :PARTMENT OF COMMERCE National 26 Bureau 5753 indards .557 


\title{
UNITED STATES DEPARTMENT OF COMMERCE \\ Maurice H. Stans, Secretary
}

NATIONAL BUREAU OF STANDARDS L Lewis M. Branscomb, Director

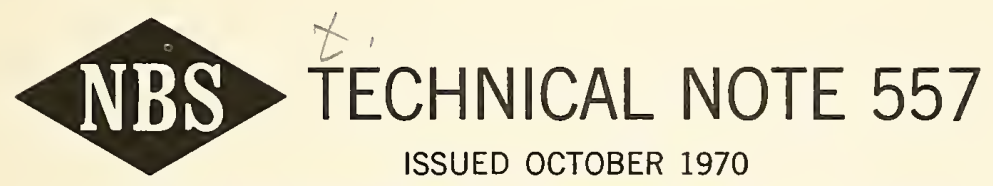

Nat. Bur. Stand. (U.S.), Tech. Note 557, 25 pages (Oct. 1970) CODEN: NBTNA

\section{The Brake Pedal Force Capability of Adult Females}

\author{
Richard W. Radlinski and James I. Price
Office of Vehicle Systems Research
Institute for Applied Technology
National Bureau of Standards
Washington, D.C. 20234

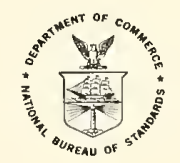

NBS Technical Notes are designed to supplement the Bureau's regular publications program. They provide a means for making available scientific data that are of transient or limited interest. Technical Notes may be listed or referred to in the open literature. 


\section{CONT ENTS}

Page

1. Introduction-... I

2. Procedure-1. 3

2. 1 Apparatus and Instrumentation-.... 3

2. 2 Test Subjects 5

2. 3 Data Acquisition-_... 6

3. Results of Test-_... 7

4. Discussion of Results_-_._.

4. 1 Test Subject Sample-1 9

4. 2 Motivational Considerations-_..-_. 9

4. 3 Pedal Force Results-_. 17

5. Conclusions-_. 23

6. References-23 
THE BRAKE PEDAL FORCE

CAPABILITY OF ADULT FEMALES*

R. W. Radlinski and J. I. Price

\section{ABSTRACT}

A survey of the brake pedal force capability of 105 women employees at the National Bureau of Standards, Washington, D. C., was performed utilizing two stationary passenger automobiles as test vehicles. Results showed that over $50 \%$ of the test subjects could not achieve an average sustained brake pedal force of 200 $1 b$, a value which is considered an acceptable braking system input force under certain conditions of the current Federal Motor Vehicle Safety Standard (FMVSS No. 105) for passenger vehicle braking system performance.

Key words: Automotive braking systems; brakes; brake pedal forces; Federal Motor Vehicle Safety Standards; pedal effort; women, strength of

\section{INTRODUCTION}

Federal Motor Vehicle Safety Standard No. 105 [1] and SAE Recommended Practice J937a, Service Brake System Performance Requirements Passenger Car [2], allow brake pedal forces up to $200 \mathrm{lb}$ under certain conditions. Since women in general are physically weaker than men, their pedal force capabilities are an important factor in establishing a maximum acceptable level for brake system input forces. There has been considerable discussion recently in government and industry as to

\footnotetext{
*This work was carried out at the National Bureau of Standards under the sponsorship of the Department of Transportation, National Highway Safety Bureau. The opinions, findings and conclusions expressed are those of the authors and not necessarily those of the National Highway Safety Bureau.
} 

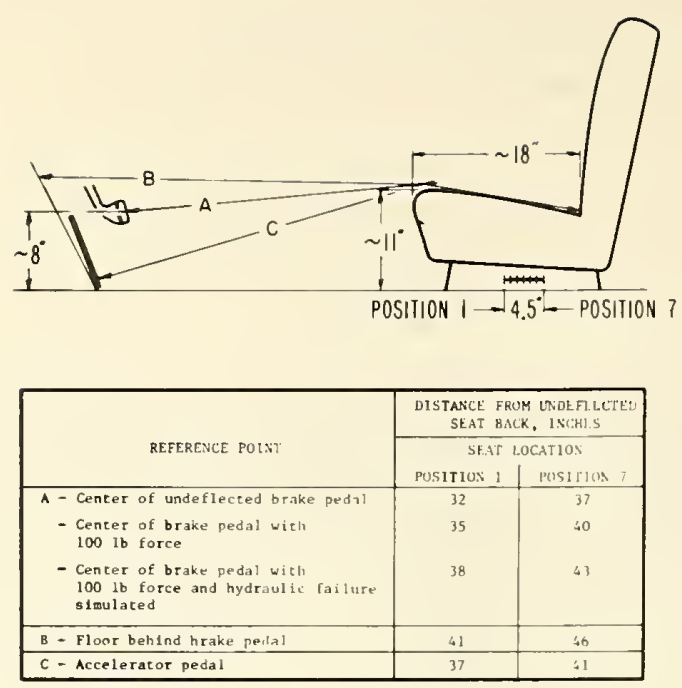

DIMENSIONS FOR VEHICLE " 4 "
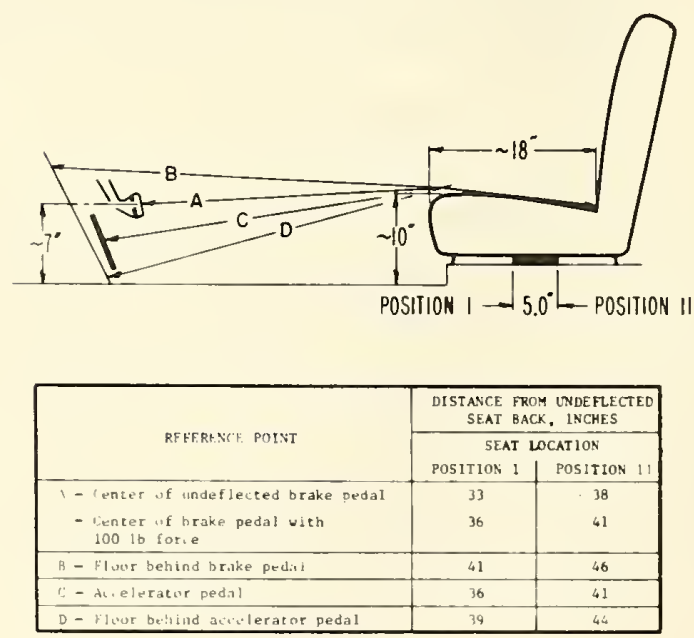

DIMENSIONS FOR VEHICLE " 8 "

\section{FIG I-DIMENSIONS OF TEST VEHICLES}


whether the $200 \mathrm{lb}$ force allowed is realistic when consideration is given to actual brake usage conditions and the pedal force capabilities of the weaker portion of the driving population.

The object of this test program was to obtain data on the brake pedal force capabilities of females of driving age. All testing was done in stationary vehicles. It is recognized that testing in a moving vehicle with simulated emergency or panic situations may have in some cases produced higher pedal efforts. However, people react differently under emergency conditions and some pedal efforts may have been lower. It is believed that the results obtained in this test program are a reasonable basis for establishing maximum pedal effort requirements.

\section{PROCEDURE}

\subsection{Apparatus and Instrumentation}

Two late model cars were used for the tests. Both vehicles were equipped with standard (non-power) brakes. The driver seat in vehicle A was of the conventional adjustable bench type and the seat in vehicle $B$ was a bucket type with fore and aft adjustment. Both contained lap type seat belts. Sketches of the brake pedal to seat relationship for the two vehicles are shown in figure 1.

Both vehicles were equipped with split hydraulic braking systems. The split system consists of two separate hydraulic circuits operated by tandem pistons in a common master cylinder. One circuit actuates the front brakes and the other actuates the rear brakes. In the event of a leak or rupture in one circuit, the other circuit remains operational. However, because of the construction of the master cylinder, brake pedal travel is increased when fluid loss occurs. A hydraulic system failure therefore is accompanied by an increase in the distance between the seat and brake pedal with the brake in the applied position. To evaluate the effect of increased pedal travel on maximum pedal force capabilities a failure simulated system was installed in vehicle A. The system, diagrammed in figure 2 , consists of a solenoid operated 


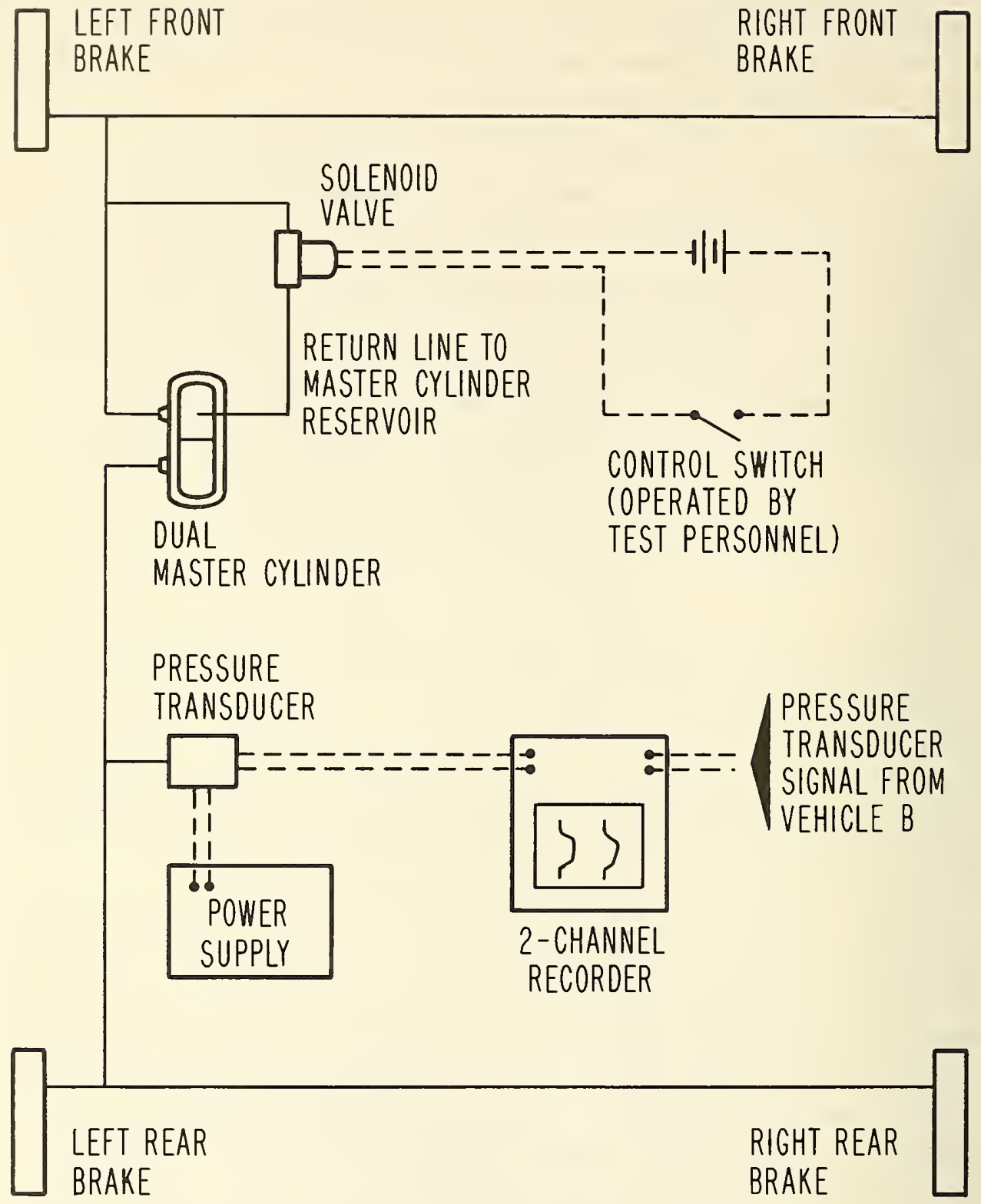

FIG 2-SCHEMATIC OF BRAKE HYDRAULIC SYSTEM IN VEHICLE "A" SHOWING INSTRUMENTATION AND FAILURE SIMULATION SYSTEM 
valve which when energized returns displaced fluid in the front hydraulic circuit to the master cylinder reservoir. The simulated failure caused an increase of about 3 inches in pedal travel at a pedal force of 100 pounds.

Effective pedal forces were obtained by measuring fluid pressure in the brake systems. The instrumentation is shown schematically in figure 2. This system consisted of a pressure transducer for each vehicle, a dc power supply and a 2-channel recorder. The transducer provided an electrical output proportional to pressure which was recorded versus time on the recorder. With the non-power brakes there was a linear relationship between pedal force and line pressure and the system could be calibrated to read directly in pedal force. This was achieved by using a pedal force transducer attached to the brake pedal to indicate force applied. A known force was applied normal to the brake pedal pad and the recorder gain control was adjusted to produce the desired span on the pedal force axis.

Position of the driver's seat during the test was considered to be a possibly important factor for use in analyzing results. A simple pointer and scale device was installed in each vehicle to indicate seat position. The seat in vehicle $A$ was adjustable to seven equally spaced positions over a distance of 4.5 inches. The seat in vehicle $B$ was adjustable to eleven equally spaced positions over a distance of 5 inches.

\subsection{Test Subjects}

Test subjects were obtained on a volunteer basis and consisted of female persons, the great majority of whom were employed at the National Bureau of Standards. They ranged in height from 59 to 69 inches, in weight from 102 to 206 pounds and in age from 18 to 62 years. The test group was of working age and thus no older persons were included. 


\subsection{Data Acquisition}

Each subject was informed before entering the vehicle that the purpose of the test was to determine how hard she could push on the brake pedal. Each subject, except number 5, was first tested in vehicle $A$ and then vehicle B. Four brake applications were made in vehicle A; two with the system intact and two with a simulated failure. Two brake applications were made in vehicle $B$. It was thought that as short a time as practicable should be used for each application since test subject fatigue could affect the results of the later applications. A11 applications were maintained for approximately five seconds. This interval was chosen because it is representative of the approximate time required to bring a vehicle to a complete stop from $60 \mathrm{mph}$ in a panic situation. Pedal force versus time was recorded on a chart recorder for al1 applications.

The tests were conducted as follows:

(a) Brake Application Number 1 - The subject was asked to sit in vehicle $A$, adjust the seat to a comfortable driving position and fasten the seat belt. The tester noted the seat position on an information card. The seat was not moved by the individual test subject after the initial adjustment. The subject was asked to push as hard as possible on the brake pedal until told to release it. After a five second interval she was told to release the pedal in this and all subsequent applications.

(b) Brake Application Number 2 - The subject was asked to push again and encouraged to try to push harder until told to release.

(c) Brake Application Number 3 - The subject was told that pedal travel was going to be increased (at this point the solenoid valve was opened, simulating a system failure) and to push again as hard as possible until told to release.

(d) Brake Application Number 4 - The subject was asked to push again (with the simulated failure existing) and encouraged to try to push harder. 
(e) Brake Application Number 5 - The subject was asked to leave vehicle $A$ and sit in vehicle $B$. The instructions to adjust the seat to a comfortable driving position and fasten the seat belt were repeated and seat position was noted. The subject was asked to push as hard as possible on the brake pedal until told to release.

(f) Brake Application Number 6 - The subject was asked to push again and encouraged to try to push harder until told to release.

The subject was informed that the test was complete and requested to fill out an information card giving height, weight, age and occupation. The subject dropped the card into a box and was not requested to give her name or show the card to testing personnel. This was an attempt to eliminate any hesitation on the part of the subject to provide this information. Information cards were related to recorder charts by assigning a test number to each subject. A sample information card is shown in figure 3 .

\section{RESULTS OF TEST}

A sample recorder trace for a single brake pedal application is shown in figure 4. This sample is marked to clarify its meaning. For each subject there are six traces of this type since six pedal applications were made. For each trace two pieces of data were tabulated for analysis; the maximum pedal force reached during the application and the average pedal force sustained for a five second interval. The average sustained pedal force was obtained by a simple graphical integration approximation. This consisted of positioning a straight line with a length representing 5 seconds parallel to the time axis and intersecting the pedal force trace so that the area between the line and trace above the line is estimated to equal the area between the line and the trace below the line. The point of intersection of the line and trace represents the average pedal force sustained for five seconds. Results of applications 2, 4 and 6 are summarized in table 1 below. 


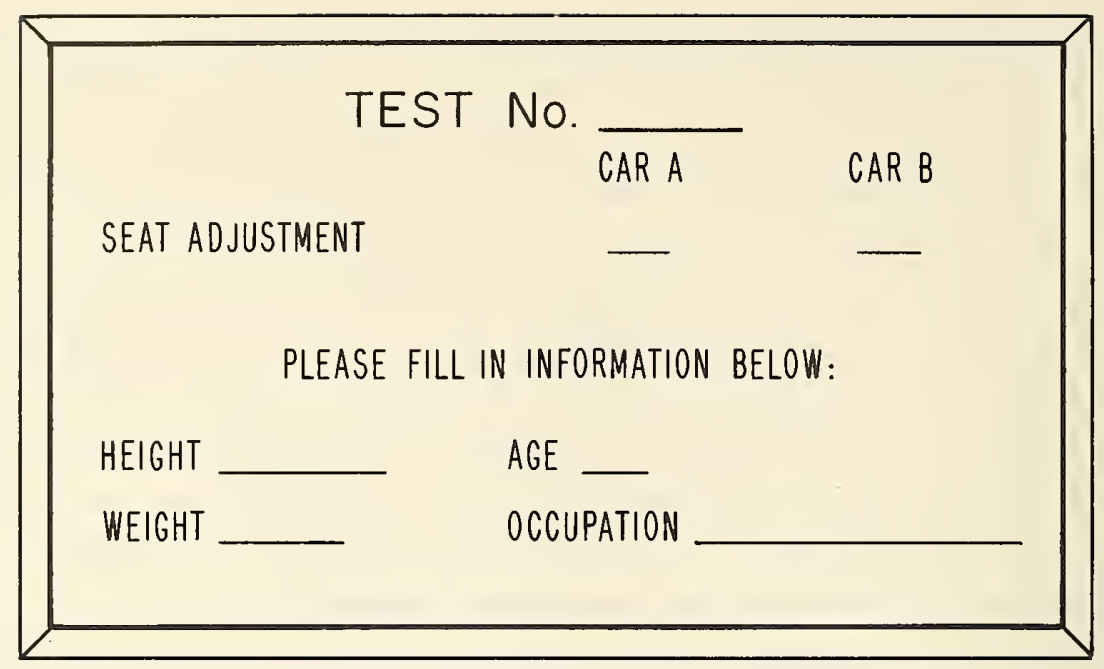

FIG 3-SAMPLE SUBJECT INFORMATION CARD

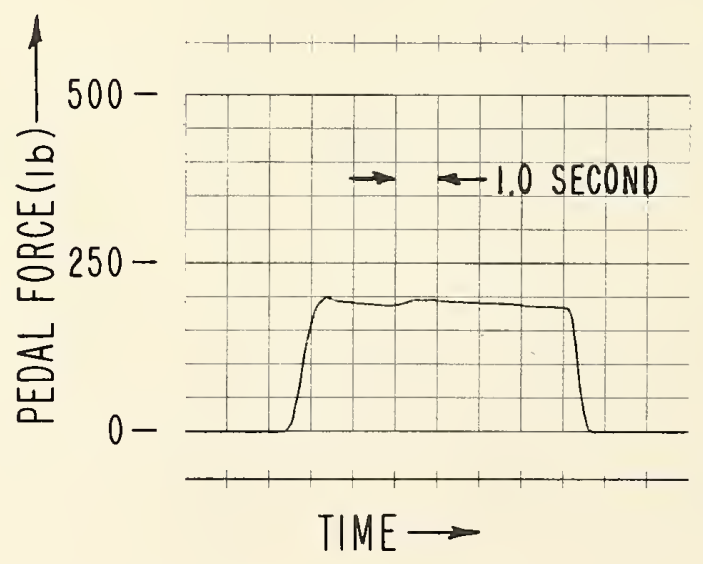

FIG 4-SAMPLE RECORDER TRACE FOR A SINGLE BRAKE PEDAL APPLICATION 
TABLE 1. AVERAGE SUSTAINED PEDAL FORCE FOR 5 SECONDS FOR SELECTED PERCENTILES

\begin{tabular}{cccc}
\hline & \multicolumn{3}{c}{ Brake application number } \\
\cline { 2 - 4 } Percentile & $\begin{array}{c}\text { Vehicle A } \\
\text { normal pedal }\end{array}$ & $\begin{array}{c}\text { Vehicle A } \\
\text { low pedal }\end{array}$ & $\begin{array}{c}\text { Vehicle B } \\
\text { normal peda1 }\end{array}$ \\
\hline \multicolumn{4}{c}{ Pedal } \\
force, pounds \\
95 & 260 & 200 & 275 \\
90 & 240 & 180 & 250 \\
80 & 220 & 160 & 235 \\
50 & 175 & 130 & 190 \\
20 & 135 & 100 & 165 \\
10 & 120 & 80 & 135 \\
5 & 90 & 65 & 125 \\
\hline
\end{tabular}

Data from the subject information cards is shown in table 2. Maximum and average pedal force data for each of the six pedal applications are shown in table 3 .

\section{DISCUSSION OF RESULTS}

\subsection{Test Subject Sample}

Frequency distributions (histograms) of height, weight and age of test subjects are shown in figure 5. Comparison of this data to information in Reference 1 of a random sampling of 58,343 women 18-79 years of age indicates that the present sample of test subjects contained no serious distortions.

\subsection{Motivationa1 Considerations}

There are many factors which might affect the pedal force capabilities of drivers in the stationary test situation as well as while driving when an emergency occurs. Some factors difficult to assess in a test series are motivation and cooperation of the test subjects and 

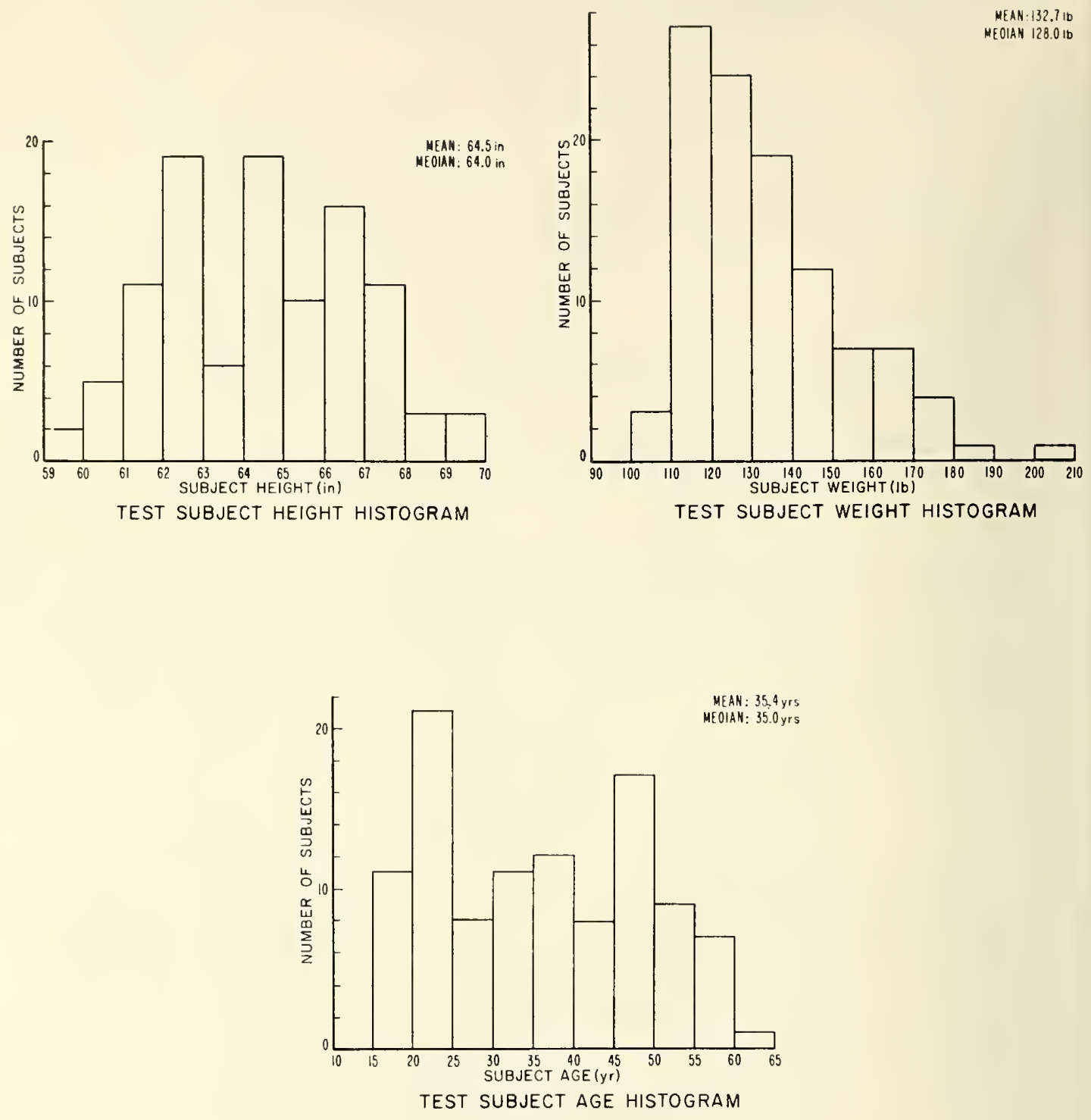

FIG 5-TEST SUBJECT INFORMATION HISTOGRAMS 
TABLE 2.. DESCRIPTION OF TEST SUBJECTS AND SEAT POSITIONS

\begin{tabular}{|c|c|c|c|c|c|c|}
\hline $\begin{array}{l}\text { Subject } \\
\text { number }\end{array}$ & $\begin{array}{c}\text { Height, } \\
\text { in }\end{array}$ & $\begin{array}{c}\text { Weight, } \\
1 \mathrm{~b}\end{array}$ & Age & Occupation & $\begin{array}{l}\text { Seat } \\
\text { position, } \\
\text { Vehicle A }\end{array}$ & $\begin{array}{c}\text { Seat } \\
\text { position, } \\
\text { Vehicle B }\end{array}$ \\
\hline $1^{a}$ & 61 & 110 & 18 & Secretary & 1 & 1 \\
\hline 2 & 59 & 125 & 35 & Cafeteria & 2 & 1 \\
\hline 3 & 65 & 112 & 19 & Typist & 2 & 2 \\
\hline 4 & 63 & 130 & 18 & Typist & 2 & 1 \\
\hline 5 & 64 & 110 & 21 & Typist & 1 & 1 \\
\hline 6 & 67 & 175 & 30 & Cafeteria & 3 & 3 \\
\hline 7 & 67 & 139 & 43 & Admin. Aid & 5 & 6 \\
\hline 8 & 64 & 135 & 23 & Secretary & 3 & 1 \\
\hline 9 & 65 & 121 & 18 & Typist & 5 & 6 \\
\hline 10 & 61 & 128 & 48 & Clerk & 2 & 1 \\
\hline 11 & 64 & 158 & 48 & Cafeteria & 3 & 5 \\
\hline 12 & 66 & 115 & 20 & Typist & 3 & 3 \\
\hline 13 & 62 & 135 & 25 & Typist & 1 & 1 \\
\hline 14 & 67 & 185 & 53 & Clerk & 4 & 5 \\
\hline 15 & 63 & 115 & 59 & Cafeteria & 2 & 5 \\
\hline 16 & 68 & 150 & 20 & Secretary & 4 & 5 \\
\hline 17 & 62 & 140 & 28 & Typist & 4 & 2 \\
\hline 18 & 62 & 115 & 33 & Clerk & 2 & 1 \\
\hline 19 & 60.5 & 102 & 46 & Typist & 2 & 1 \\
\hline 20 & 64 & 110 & 18 & (Not given) & 2 & 1 \\
\hline 21 & 62.5 & 125 & 39 & Clerk & 2 & 1 \\
\hline 22 & 67 & 160 & 58 & Clerk & 5 & 8 \\
\hline 23 & 64 & 115 & 26 & Comp. Prog. & 3 & 2 \\
\hline 24 & 67 & 163 & 46 & Housewife & 3 & 4 \\
\hline 25 & 60.5 & 110 & 38 & Lib. Tech. & 1 & 1 \\
\hline 26 & 67 & 157 & 43 & Clerk & 5 & 7 \\
\hline 27 & 63 & 118 & 19 & Clerk & 1 & 1 \\
\hline 28 & 61 & 120 & 24 & Supervisor & 1 & 1 \\
\hline 29 & 62 & 130 & 47 & Clerk & 1 & 1 \\
\hline 30 & 64 & 125 & 22 & Clerk & 3 & 2 \\
\hline 31 & 64 & 169 & 47 & (Not given) & 3 & 2 \\
\hline 32 & 65 & 150 & 34 & Clerk & 1 & 3 \\
\hline 33 & 62 & 140 & 49 & Analyst & 1 & 1 \\
\hline 34 & 66 & 140 & 38 & Clerk & 4 & 6 \\
\hline 35 & 64 & 116 & 23 & Secretary & 2 & 3 \\
\hline
\end{tabular}

a Used left foot

(Continued) 


\begin{tabular}{|c|c|c|c|c|c|c|}
\hline $\begin{array}{l}\text { Subject } \\
\text { number }\end{array}$ & $\begin{array}{l}\text { Height, } \\
\text { in }\end{array}$ & $\begin{array}{c}\text { Weight, } \\
1 \mathrm{~b}\end{array}$ & Age & Occupation & $\begin{array}{l}\text { Seat } \\
\text { position, } \\
\text { Vehicle A }\end{array}$ & $\begin{array}{c}\text { Seat } \\
\text { position, } \\
\text { Vehicle B }\end{array}$ \\
\hline 36 & 64 & 150 & 54 & Typist & 4 & 7 \\
\hline 37 & 62 & 135 & 56 & Accountant & 4 & 6 \\
\hline 38 & 64 & 114 & 19 & Secretary & 2 & 1 \\
\hline 39 & 61 & 114 & 30 & Secretary & 2 & 3 \\
\hline 40 & 61 & 125 & 30 & Comp. Prog. & 1 & 1 \\
\hline $41^{a}$ & 66 & 120 & 42 & Analyst & 6 & 8 \\
\hline 42 & 67.5 & 140 & 54 & Analyst & 4 & 4 \\
\hline 43 & 62.5 & 119 & 20 & Clerk & 1 & 1 \\
\hline 44 & 64 & 124 & 21 & Clerk & 4 & 6 \\
\hline 45 & 64 & 125 & 37 & Clerk & 2 & 1 \\
\hline 46 & 60 & 110 & 40 & Chemist & 2 & 1 \\
\hline 47 & 66 & 170 & 45 & Physicist & 5 & 9 \\
\hline 48 & 66 & 138 & 49 & Adm. Asst. & 5 & 7 \\
\hline 49 & 62 & 118 & 48 & Typist & 3 & 1 \\
\hline 50 & 69 & 165 & 50 & (Not given) & 3 & 7 \\
\hline 51 & 59 & 125 & 62 & Acct. Tech. & 3 & 7 \\
\hline 52 & 63 & 115 & 32 & Supervisor & 1 & 1 \\
\hline 53 & 64 & 109 & 21 & Clerk & 3 & 5 \\
\hline 54 & 66 & 130 & 38 & Physicist & 5 & 7 \\
\hline 55 & 61 & 128 & 49 & Math. Tech. & 1 & 1 \\
\hline 56 & 62 & 137 & 20 & Bookkeeper & 2 & 1 \\
\hline 57 & 67 & 126 & 20 & Secretary & 7 & 7 \\
\hline 58 & 66 & 133 & 19 & Clerk & 2 & 4 \\
\hline 59 & 64 & 142 & 31 & Supervisor & 3 & 4 \\
\hline 60 & 61.75 & 125 & 20 & Typist & 1 & 1 \\
\hline 61 & 61 & 135 & 25 & Clerk & 1 & 1 \\
\hline 62 & 64 & 160 & 22 & Secretary & 3 & 4 \\
\hline 63 & 62.5 & 117 & 42 & (Not given) & 1 & 1 \\
\hline 64 & 63 & 115 & 20 & Secretary & 1 & 1 \\
\hline 65 & 65 & 115 & 23 & Personnel & 3 & 2 \\
\hline 66 & 67.5 & 146 & 20 & Clerk & 4 & 6 \\
\hline 67 & 64 & 150 & 55 & Personne1 & 4 & 2 \\
\hline 68 & 64 & 140 & 53 & Procurement & 4 & 2 \\
\hline 69 & 62 & 115 & 25 & Adm. Aid & 1 & 2 \\
\hline 70 & 67 & 135 & 36 & Tech.Info.Sp & 3 & 2 \\
\hline
\end{tabular}

a Used left foot 
TABLE 2 (Cont'd)

\begin{tabular}{|c|c|c|c|c|c|c|}
\hline $\begin{array}{l}\text { Subject } \\
\text { number }\end{array}$ & $\begin{array}{c}\text { Height, } \\
\text { in }\end{array}$ & $\begin{array}{c}\text { Weight, } \\
1 \mathrm{~b}\end{array}$ & Age & Occupation & $\begin{array}{c}\text { Seat } \\
\text { position, } \\
\text { Vehicle A }\end{array}$ & $\begin{array}{c}\text { Seat } \\
\text { position, } \\
\text { Vehicle B }\end{array}$ \\
\hline 71 & 66.5 & 122 & 41 & Secretary & 4 & 4 \\
\hline 72 & 61 & 116 & 45 & Secretary & 1 & 1 \\
\hline 73 & 61 & 120 & 56 & Secretary & 1 & 1 \\
\hline 74 & 63 & 145 & 27 & Secretary & 4 & 1 \\
\hline 75 & 61 & 110 & 43 & Secretary & 1 & 1 \\
\hline 76 & 69 & 149 & 25 & Physicist & 7 & 9 \\
\hline 77 & 62 & 115 & 34 & Clerk & 1 & 1 \\
\hline 78 & 68 & 158 & 18 & Secretary & 7 & 7 \\
\hline 79 & 62 & 137 & 48 & Laborer & 2 & 4 \\
\hline 80 & 66 & 176 & 55 & Laborer & 3 & 6 \\
\hline 81 & 68 & 135 & 51 & Budget Analyst & 5 & 7 \\
\hline 82 & 65.5 & 160 & 27 & Key Punch & 2 & 1 \\
\hline 83 & 65 & 140 & 46 & Adm. Asst. & 4 & 7 \\
\hline 84 & 62 & 113 & 31 & Personnel & 1 & 1 \\
\hline 85 & 66.5 & 125 & 20 & Clerk & 4 & 6 \\
\hline 86 & 65 & 108 & 30 & Statistician & 3 & 3 \\
\hline 87 & 66 & 125 & 51 & Physicist & 3 & 1 \\
\hline 88 & 65 & 130 & 21 & Clerk & 3 & 3 \\
\hline 89 & 69 & 140 & 38 & Physicist & 4 & 6 \\
\hline 90 & 67 & 206 & 36 & Personne1 & 6 & 7 \\
\hline 91 & 62 & 125 & 38 & Personne1 & 2 & 1 \\
\hline 92 & 66 & 120 & 48 & Clerk & 3 & 5 \\
\hline 93 & 61.5 & 124 & 48 & Sys. Analyst & 1 & 1 \\
\hline 94 & 66 & 165 & 51 & Mgmt. Analyst & 5 & 8 \\
\hline 95 & 60 & 134 & 36 & Secretary & 2 & 3 \\
\hline 96 & 65 & 135 & 22 & Clerk & 2 & 3 \\
\hline 97 & 64 & 130 & 53 & Physicist & 2 & 2 \\
\hline $98^{\mathrm{a}}$ & 65 & 140 & 34 & Statistician & 6 & 7 \\
\hline 99 & 64 & 175 & 23 & Mathematician & 4 & 4 \\
\hline 100 & 66 & 119 & 19 & Typist & 4 & 4 \\
\hline 101 & 62 & 125 & 17 & Clerk & 2 & 1 \\
\hline 102 & 66 & 110 & 37 & R.N. & 3 & - \\
\hline 103 & 66 & 130 & 55 & Budget Analyst & 6 & 8 \\
\hline 104 & 60 & 128 & 41 & Mathematician & 1 & 1 \\
\hline 105 & 62.5 & 128 & 48 & Info. Spec. & 4 & 3 \\
\hline
\end{tabular}

$a_{\text {Used }}$ left foot 


\begin{tabular}{|c|c|c|c|c|c|c|c|c|c|c|c|c|}
\hline \multirow{4}{*}{$\begin{array}{l}\text { Subject } \\
\text { number }\end{array}$} & \multicolumn{12}{|c|}{ Brake Application Number } \\
\hline & \multicolumn{8}{|c|}{ Vehicle A } & \multicolumn{4}{|c|}{ Vebicle B } \\
\hline & \multicolumn{2}{|c|}{1} & \multicolumn{2}{|c|}{2} & \multicolumn{2}{|c|}{3} & \multicolumn{2}{|c|}{4} & \multicolumn{2}{|c|}{5} & \multicolumn{2}{|c|}{6} \\
\hline & Max & $\mathrm{Av}$ & Max & $\mathrm{Av}$ & Ma: & Av & Max & Av & $\operatorname{Max}$ & $\mathrm{Av}$ & $\operatorname{Max}$ & $\mathrm{Av}$ \\
\hline & & & & & & edal & e, $\mathrm{pc}$ & unds & & & & \\
\hline 1 & 165 & 145 & 150 & 140 & 65 & 60 & 100 & 85 & 130 & 120 & 145 & 135 \\
\hline 2 & 140 & 130 & 200 & 180 & 65 & 60 & 135 & 120 & 150 & 130 & 195 & 175 \\
\hline 3 & 185 & 180 & 245 & 230 & 175 & 160 & 155 & 145 & 220 & 205 & 215 & 210 \\
\hline 4 & 280 & 250 & 290 & 250 & 220 & 175 & 180 & 170 & 240 & 220 & 255 & 235 \\
\hline 5 & 225 & 220 & 235 & 220 & 165 & 150 & 170 & 150 & 165 & 150 & 245 & 230 \\
\hline 6 & 160 & 140 & 260 & 235 & 190 & 180 & 195 & 170 & 200 & 195 & 265 & 235 \\
\hline 7 & 130 & 115 & 165 & 140 & 120 & 110 & 125 & 115 & 150 & 145 & 200 & 185 \\
\hline 8 & 110 & 105 & 195 & 180 & 95 & 80 & 115 & 85 & 160 & 135 & 145 & 135 \\
\hline 9 & 115 & 110 & 180 & 160 & 95 & 90 & 125 & 110 & 185 & 165 & 180 & 170 \\
\hline 10 & 140 & 110 & 175 & 135 & 75 & 50 & 95 & 60 & 185 & 160 & 190 & 170 \\
\hline 11 & 145 & 115 & 170 & 140 & 110 & 105 & 120 & 110 & 180 & 160 & 190 & 160 \\
\hline 12 & 175 & 165 & 210 & 190 & 200 & 180 & 225 & 210 & 245 & 235 & 260 & 250 \\
\hline 13 & 220 & 210 & 200 & 195 & 195 & 180 & 200 & 185 & 195 & 180 & 245 & 235 \\
\hline 14 & 225 & 200 & 240 & 200 & 200 & 170 & 190 & 155 & 210 & 175 & 240 & 195 \\
\hline 15 & 165 & 150 & 165 & 150 & 175 & 150 & 170 & 155 & 200 & 185 & 155 & 140 \\
\hline 16 & 180 & 175 & 200 & 190 & 200 & 190 & 210 & 190 & 235 & 220 & 270 & 255 \\
\hline 17 & 75 & 70 & 140 & 130 & 100 & 85 & 115 & 105 & 120 & 105 & 165 & 160 \\
\hline 18 & 120 & 115 & 150 & 135 & 70 & 65 & 85 & 80 & 90 & 75 & 105 & 100 \\
\hline 19 & 70 & 65 & 95 & 85 & 70 & 65 & 95 & 80 & 75 & 70 & 105 & 95 \\
\hline 20 & 190 & 180 & 180 & 175 & 165 & 150 & 170 & 160 & 195 & 190 & 220 & 210 \\
\hline 21 & 150 & 145 & 205 & 175 & 105 & 100 & 130 & 110 & 135 & 120 & 210 & 190 \\
\hline 22 & 210 & 195 & 240 & 230 & 145 & 140 & 165 & 155 & 190 & 180 & 210 & 200 \\
\hline 23 & 140 & 120 & 210 & 185 & 130 & 100 & 145 & 115 & 170 & 150 & 175 & 140 \\
\hline 24 & 100 & 85 & 170 & 160 & 125 & 105 & 150 & 120 & 165 & 145 & 220 & 210 \\
\hline 25 & 165 & 155 & 185 & 180 & 150 & 145 & 160 & 145 & 180 & 175 & 210 & 200 \\
\hline 26 & 230 & 225 & 265 & 260 & 180 & 140 & 170 & 150 & 230 & 225 & 225 & 215 \\
\hline 27 & 235 & 210 & 270 & 260 & 190 & 170 & 185 & 175 & 220 & 200 & 250 & 245 \\
\hline 28 & 190 & 180 & 210 & 205 & 110 & 100 & 115 & 100 & 185 & 160 & 210 & 205 \\
\hline 29 & 180 & 160 & 220 & 180 & 100 & 90 & 145 & 130 & 165 & 145 & 205 & 170 \\
\hline 30 & 100 & 90 & 100 & 95 & 85 & 50 & 80 & 65 & 150 & 120 & 145 & 135 \\
\hline 31 & 180 & 165 & 235 & 230 & 160 & 155 & 170 & 160 & 220 & 215 & 260 & 255 \\
\hline 32 & 155 & 145 & 255 & 240 & 130 & 130 & 210 & 200 & 265 & 255 & 265 & 260 \\
\hline 33 & 180 & 80 & 150 & 120 & 130 & 80 & 105 & 95 & 125 & 120 & 220 & 185 \\
\hline 34 & 115 & 110 & 190 & 175 & 90 & 80 & 115 & 100 & 165 & 160 & 175 & 165 \\
\hline 35 & 160 & 150 & 160 & 140 & 105 & 95 & 65 & 65 & 200 & 190 & 215 & 200 \\
\hline
\end{tabular}


TABLE 3 (Cont'd)

\begin{tabular}{|c|c|c|c|c|c|c|c|c|c|c|c|c|}
\hline \multirow{4}{*}{$\begin{array}{l}\text { Subject } \\
\text { number }\end{array}$} & \multicolumn{12}{|c|}{ Brake Application Number } \\
\hline & \multicolumn{8}{|c|}{ Vechicle A } & \multicolumn{4}{|c|}{ Vehicle B } \\
\hline & \multicolumn{2}{|c|}{1} & \multicolumn{2}{|c|}{2} & \multicolumn{2}{|c|}{3} & \multicolumn{2}{|c|}{4} & \multicolumn{2}{|c|}{5} & \multicolumn{2}{|c|}{6} \\
\hline & $\operatorname{Max}$ & $\mathrm{Av}$ & $\operatorname{Max}$ & $\mathrm{Av}$ & \multirow{2}{*}{\multicolumn{4}{|c|}{$\begin{array}{rc}\text { Max Av } & \text { Max Av } \\
\text { Pedal force, pounds }\end{array}$}} & $\operatorname{Max}$ & $\mathrm{Av}$ & $\operatorname{Max}$ & $\mathrm{Av}$ \\
\hline & & & & & & & & & & & & \\
\hline 36 & 200 & 180 & 225 & 205 & 145 & 120 & $145^{5}$ & 120 & 210 & 190 & 190 & 180 \\
\hline 37 & 170 & 145 & 155 & 135 & 105 & 85 & 130 & 95 & 180 & 155 & 205 & 180 \\
\hline 38 & 150 & 145 & 140 & 135 & 90 & 85 & 90 & 85 & 130 & 125 & 135 & 125 \\
\hline 39 & 185 & 180 & 225 & 190 & 130 & 120 & 135 & 100 & 190 & 175 & 240 & 210 \\
\hline 40 & 115 & 110 & 175 & 155 & 120 & 110 & 115 & 95 & 185 & 180 & 195 & 180 \\
\hline 41 & 125 & 110 & 100 & 90 & 65 & 60 & 60 & 50 & 125 & 115 & 160 & 145 \\
\hline 42 & 185 & 180 & 250 & 245 & 160 & 155 & 165 & 155 & 230 & 220 & 250 & 240 \\
\hline 43 & 90 & 85 & 115 & 100 & 125 & 110 & 130 & 115 & 160 & 130 & 145 & 135 \\
\hline 44 & 125 & 115 & 155 & 140 & 105 & 95 & 120 & 105 & 140 & 135 & 190 & 175 \\
\hline 45 & 70 & 60 & 90 & 90 & 105 & 80 & 130 & 100 & 110 & 80 & 160 & 135 \\
\hline 46 & 135 & 120 & 175 & 160 & 95 & 75 & 80 & 65 & 145 & 140 & 170 & 165 \\
\hline 47 & 145 & 145 & 160 & 155 & 150 & 145 & 150 & 125 & 160 & 155 & 190 & 185 \\
\hline 48 & 150 & 145 & 185 & 175 & 120 & 105 & 140 & 140 & 175 & 160 & 185 & 175 \\
\hline 49 & 130 & 120 & 165 & 150 & 110 & 100 & 135 & 115 & 160 & 150 & 175 & 165 \\
\hline 50 & 185 & 155 & 150 & 120 & 125 & 120 & 145 & 140 & 170 & 160 & 185 & 165 \\
\hline 51 & 135 & 130 & 160 & 145 & 70 & 65 & 110 & 95 & 135 & 130 & 165 & 150 \\
\hline 52 & 130 & 130 & 180 & 175 & 110 & 100 & 160 & 130 & 195 & 180 & 185 & 170 \\
\hline 53 & 155 & 120 & 155 & 120 & 90 & 75 & 105 & 80 & 195 & 170 & 200 & 170 \\
\hline 54 & 110 & 100 & 135 & 130 & 155 & 140 & 155 & 150 & 190 & 185 & 200 & 190 \\
\hline 55 & 120 & 115 & 150 & 145 & 130 & 115 & 150 & 135 & 205 & 195 & 220 & 205 \\
\hline 56 & 160 & 145 & 190 & 185 & 125 & 125 & 140 & 130 & 200 & 195 & 215 & 210 \\
\hline 57 & 195 & 190 & 225 & 220 & 155 & 150 & 190 & 185 & 220 & 210 & 220 & 220 \\
\hline 58 & 115 & 105 & 125 & 115 & 120 & 115 & 145 & 130 & 140 & 130 & 185 & 170 \\
\hline 59 & 150 & 125 & 185 & 175 & 120 & 100 & 145 & 130 & 155 & 140 & 215 & 190 \\
\hline 60 & 170 & 165 & 200 & 195 & 150 & 145 & 170 & 155 & 210 & 200 & 230 & 215 \\
\hline 61 & 110 & 105 & 95 & 90 & 110 & 100 & 120 & 110 & 195 & 185 & 230 & 220 \\
\hline 62 & 260 & 235 & 320 & 290 & 250 & 240 & 235 & 210 & 320 & 305 & 330 & 310 \\
\hline 63 & 250 & 235 & 295 & 275 & 220 & 210 & 260 & 250 & 340 & 320 & 310 & 300 \\
\hline 64 & 190 & 170 & 215 & 180 & 215 & 205 & 210 & 205 & 260 & 240 & 260 & 240 \\
\hline 65 & 105 & 100 & 175 & 165 & 90 & 85 & 130 & 120 & 210 & 200 & 245 & 235 \\
\hline 66 & 175 & 170 & 240 & 235 & 50 & 45 & 155 & 150 & 215 & 210 & 250 & 240 \\
\hline 67 & 100 & 95 & 150 & 145 & 140 & 130 & 125 & 120 & 110 & 105 & 175 & 170 \\
\hline 68 & 175 & 160 & 180 & 175 & 100 & 95 & 100 & 95 & 190 & 170 & 185 & 180 \\
\hline 69 & 220 & 205 & 220 & 205 & 130 & 120 & 120 & 115 & 175 & 170 & 210 & 195 \\
\hline 70 & 245 & 230 & 250 & 240 & 185 & 180 & 190 & 180 & 265 & 250 & 285 & 275 \\
\hline
\end{tabular}

(Continued) 


\begin{tabular}{|c|c|c|c|c|c|c|c|c|c|c|c|c|}
\hline \multirow{4}{*}{$\begin{array}{l}\text { Subject } \\
\text { number }\end{array}$} & \multicolumn{12}{|c|}{ Brake Application Number } \\
\hline & \multicolumn{8}{|c|}{ Vehicle A } & \multicolumn{4}{|c|}{ Vehicle B } \\
\hline & \multicolumn{2}{|c|}{1} & \multicolumn{2}{|c|}{2} & \multicolumn{2}{|c|}{3} & \multicolumn{2}{|c|}{4} & \multicolumn{2}{|c|}{5} & \multicolumn{2}{|c|}{6} \\
\hline & $\operatorname{Max}$ & $\mathrm{Av}$ & Max & $\mathrm{Av}$ & $\operatorname{Max}$ & $\mathrm{Av}$ & Max & $\mathrm{Av}$ & $\operatorname{Max}$ & $\mathrm{Av}$ & Max & $\mathrm{Av}$ \\
\hline & & & & & Ped & al $\mathrm{fc}$ & , pou & nds & & & & \\
\hline 71 & 150 & 130 & 240 & 170 & 90 & 65 & 165 & 130 & 215 & 190 & 240 & 220 \\
\hline 72 & 180 & 175 & 200 & 190 & 115 & 105 & 135 & 130 & 135 & 130 & 150 & 145 \\
\hline 73 & 190 & 175 & 205 & 195 & 140 & 135 & 140 & 135 & 210 & 185 & 230 & 205 \\
\hline 74 & 110 & 105 & 140 & 130 & 45 & 45 & 60 & 60 & 110 & 100 & 160 & 140 \\
\hline 75 & 250 & 235 & 255 & 240 & 160 & 145 & 160 & 135 & 190 & 175 & 215 & 205 \\
\hline 76 & 215 & 205 & 285 & 270 & 210 & 195 & 180 & 175 & 280 & 250 & 285 & 275 \\
\hline 77 & 125 & 120 & 135 & 120 & 105 & 100 & 165 & 135 & 125 & 125 & 220 & 205 \\
\hline 78 & 130 & 125 & 155 & 150 & 145 & 135 & 160 & 145 & 160 & 155 & 200 & 190 \\
\hline 79 & 180 & 175 & 215 & 205 & 105 & 100 & 125 & 105 & 190 & 180 & 195 & 185 \\
\hline 80 & 195 & 195 & 270 & 260 & 175 & 165 & 175 & 170 & 250 & 230 & 230 & 225 \\
\hline 81 & 130 & 115 & 140 & 130 & 180 & 130 & 180 & 150 & 180 & 135 & 200 & 175 \\
\hline 82 & 170 & 140 & 180 & 165 & 160 & 150 & 205 & 180 & --- & --- & --- & --- \\
\hline 83 & 165 & 155 & 215 & 205 & 120 & 115 & 145 & 130 & 200 & 190 & 220 & 210 \\
\hline 84 & 225 & 200 & 300 & 250 & 220 & 180 & 240 & 220 & 270 & 230 & 285 & 250 \\
\hline 85 & 90 & 85 & 125 & 120 & 125 & 120 & 150 & 140 & 130 & 120 & 190 & 180 \\
\hline 86 & 110 & 105 & 175 & 165 & 110 & 105 & 125 & 115 & 165 & 150 & 185 & 180 \\
\hline 87 & 185 & 160 & 170 & 160 & 125 & 115 & 160 & 140 & 170 & 150 & 200 & 185 \\
\hline 88 & 170 & 160 & 180 & 175 & 165 & 145 & 150 & 140 & 195 & 185 & 205 & 195 \\
\hline 89 & 110 & 110 & 180 & 170 & 170 & 135 & 155 & 140 & 170 & 140 & 195 & 180 \\
\hline 90 & 200 & 200 & 240 & 225 & 180 & 165 & 210 & 190 & 225 & 220 & 300 & 290 \\
\hline 91 & 225 & 205 & 260 & 240 & 165 & 145 & 195 & 180 & 270 & 250 & 300 & 275 \\
\hline 92 & 150 & 140 & 190 & 170 & 140 & 135 & 185 & 170 & 210 & 200 & 225 & 220 \\
\hline 93 & 85 & 80 & 130 & 120 & 70 & 65 & 110 & 100 & 90 & 85 & 140 & 125 \\
\hline 94 & 115 & 105 & 170 & 165 & 140 & 110 & 140 & 110 & 155 & 145 & 200 & 180 \\
\hline 95 & 195 & 185 & 200 & 185 & 120 & 115 & 145 & 140 & 180 & 175 & 205 & 190 \\
\hline 96 & 170 & 165 & 200 & 180 & 180 & 155 & 185 & 180 & 180 & 165 & 155 & 150 \\
\hline 97 & 180 & 180 & 195 & 175 & 150 & 135 & 155 & 150 & 220 & 210 & 225 & 220 \\
\hline 98 & 150 & 135 & 200 & 190 & 125 & 110 & 120 & 110 & 180 & 165 & 200 & 185 \\
\hline 99 & 140 & 100 & 155 & 140 & 105 & 85 & 145 & 140 & 155 & 120 & 180 & 170 \\
\hline 100 & 95 & 95 & 150 & 135 & 105 & 95 & 145 & 120 & 125 & 120 & 155 & 145 \\
\hline 101 & 235 & 225 & 250 & 235 & 190 & 180 & 195 & 180 & 285 & 270 & 305 & 295 \\
\hline 102 & 85 & 80 & 85 & 85 & 75 & 60 & 70 & 70 & 80 & 75 & 95 & 90 \\
\hline 103 & 165 & 165 & 165 & 165 & 130 & 120 & 140 & 130 & 205 & 195 & 245 & 230 \\
\hline 104 & 115 & 115 & 195 & 180 & 120 & 110 & 90 & 90 & 170 & 155 & 210 & 190 \\
\hline 105 & 205 & 180 & 240 & 195 & 130 & 120 & 150 & 140 & 250 & 210 & 280 & 240 \\
\hline
\end{tabular}


how well they understand and carry out the instructions. Drivers are conditioned to pushing on the brake pedal up to a certain force level under normal driving conditions and may have no understanding of what force level might be required under unusual circumstances.

Some test subjects used unusual techniques to obtain maximum pedal force. Some braced their shoulders against the back of the seat and others pulled on the steering wheel. All subjects were restrained by a lap belt which, when properly fastened, would reduce the effectiveness of these techniques. Subjects were not instructed as to rate of force application. Some preferred to "slam" on the pedal while others applied the force more gradually. It has been suggested that force acting on the pedal in a decelerating vehicle might be higher than that measured in a static situation for a given driver due to additional force developed as a result of the inertia of the driver's body mass. This might be true if the driver's leg was straight and acted like a rigid link in compression. However, it is more reasonable to assume that the leg would be bent at the knee to at least some extent and thus require muscle strength to transmit inertial force. Therefore pedal force would be limited to the same muscle capability as that measured in the static situation. In any case, inertial forces do not become significant until a very high rate of deceleration is attained.

\subsection{Pedal Force Results}

The six brake pedal applications for each of the test subjects can be broken down into three separate groups.

(a) Two applications (numbers 1 and 2) in vehicle $A$ with a normal pedal height.

(b) Two applications (numbers 3 and 4) in vehicle A with a reduced pedal height (failure simulated).

(c) Two applications (numbers 5 and 6) in vehicle $B$ with a normal pedal height. 

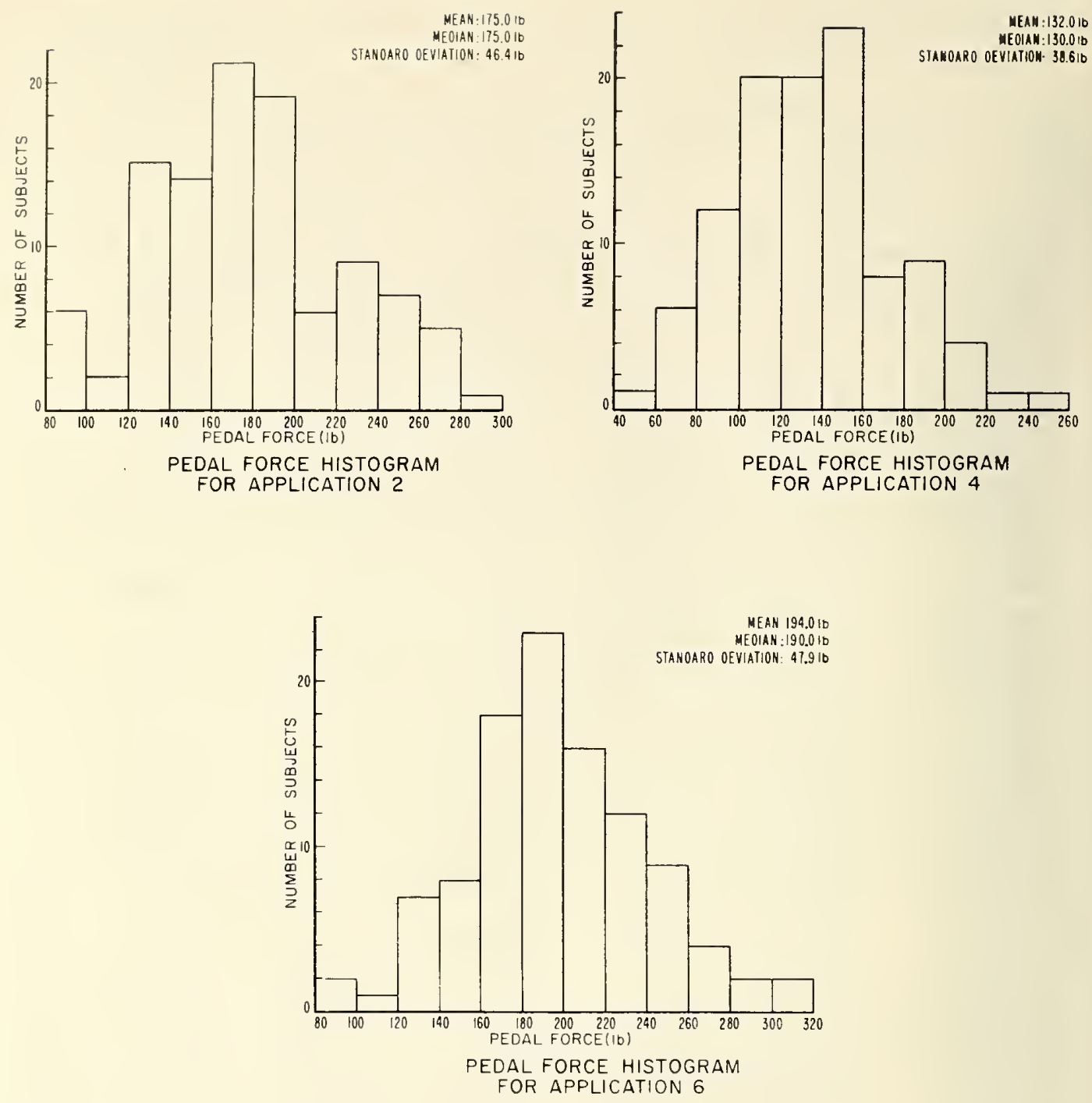

FIG 6-PEDAL FORCE HISTOGRAMS FOR THREE BRAKE APPLICATIONS 
The subject had no indication how hard she was pushing. It is believed that the second brake pedal application under each condition (numbers 2,4 and 6 ) is more indicative of the maximum capability of the test subject. The subject was more familiar with the system after the first try and with encouragement from the tester was on the average able to push harder on the second try of each group. Therefore, applications 2, 4 and 6 are of primary interest. In addition, the average pedal force sustained for five seconds during the applications is of more interest than the maximum value since the area under the forcetime curve has a direct relationship to vehicle stopping distance.

Frequency distributions (histograms) of average pedal force for applications 2, 4 and 6 are shown in figure 6. Each of these figures also shows mean, median and standard deviation calculated from the data. Histograms for maximum pedal forces would be similar in shape to those for average pedal force since plots of maximum versus average pedal force showed a linear relationship between the two values.

The histograms in figure 7 show a comparison of seat positions selected in the two vehicles. Since the number of available positions was different in each vehicle they were divided into five intervals measured from the most forward position. A similarity in the distributions of the selected positions in the two vehicles is readily apparent. As expected, further examination of the data showed a correlation between subject height and preferred seat position. Eighty-five percent of al1 subjects 63 inches in height and shorter selected seat position 1 or 2 . Twenty-five subjects (24\%) in vehicle A and forty-one (39\%) in vehicle $B$ adjusted the seat to the forwardmost position. This position was used more than any other on both cars and it could be inferred that some subjects would have moved the seat further forward if more adjustment had been available.

A comparison of the means (or medians) for applications 2 and 4 shows that reduced capability results when pedal travel is increased. Thus when the pedal moves further away from the seat the subject cannot 

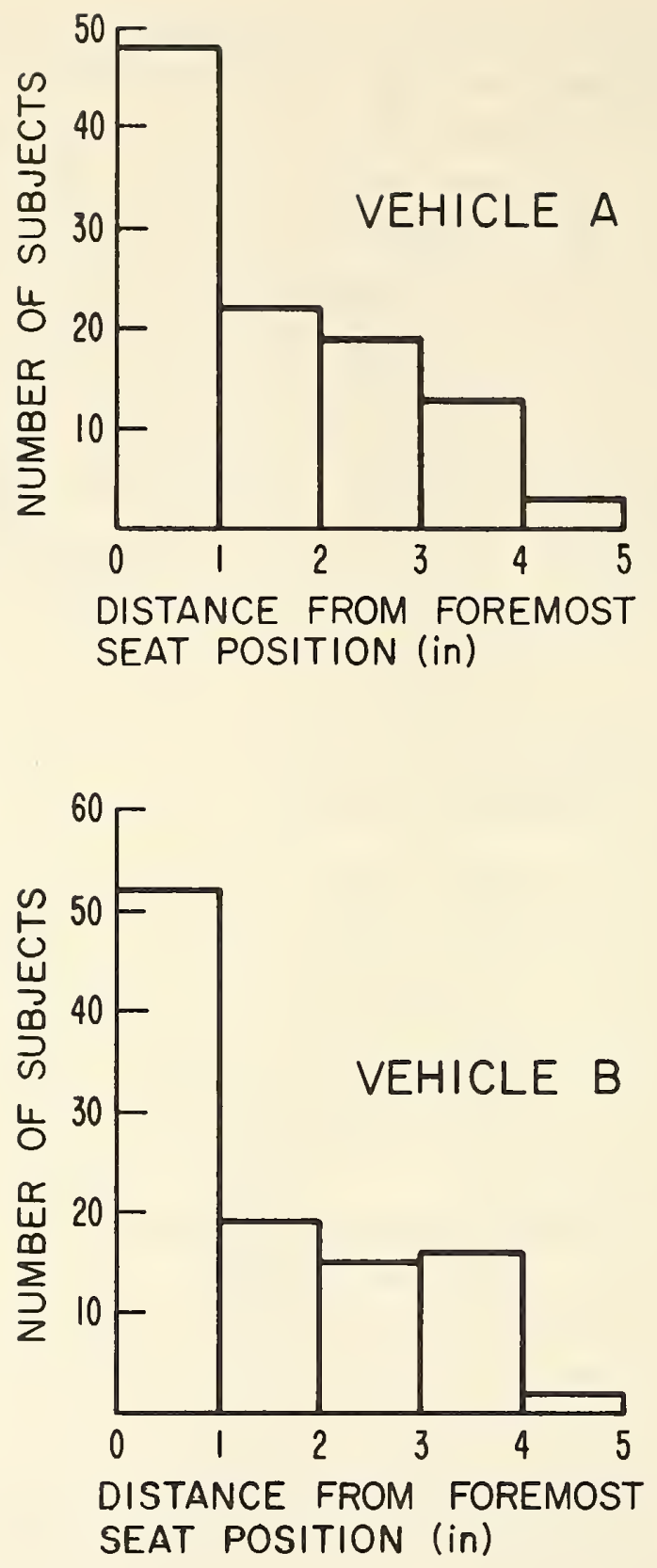

FIG 7 - SEAT ADJUSTMENT
BY TEST SUBJECTS 
push as hard and a question is raised if enough adjustment is available so that shorter subjects can reach their full capability under normal and increased pedal travel conditions. Plots of pedal force (average sustained) versus subject height were made for vehicles A and B with normal pedal travel (applications 2 and 6 respectively) and are shown in figure 8. Both plots show a wide scattering of results and indicate little, if any, relationship between subject height and pedal force capability. It does not appear then that the shorter subjects were limited by available seat positions. To investigate the increased pedal travel condition, a plot of pedal force (average sustained, application 4) versus subject height was made for vehicle A with a simulated hydraulic failure. This plot is shown in figure 8 and also indicates a wide scattering of results. The line connecting the medians for application 4 does indicate a slight upward trend in pedal force for taller subjects but the wide scattering of results prohibits any definite conclusions.

The effect of subject weight and age on pedal force capability was also investigated but no relationship was found.

A comparison of the results obtained in vehicles $A$ and $B$ with normal pedal travel indicates that subjects applied a greater force in vehicle B. For example, the median pedal force for application 2 (vehicle A) was $175 \mathrm{lb}$ while the median for application 6 (vehicle B) was $190 \mathrm{lb}$. This difference could be due to some physical difference between the vehicles and/or mental conditioning of the test subject and familiarity with the test procedure. A comparison of dimensions in figure 1 indicates little geometrical difference between vehicles; however, the seat in vehicle $B$ appeared to be firmer than the seat in vehicle $A$ and could account for some difference in subject capability. 


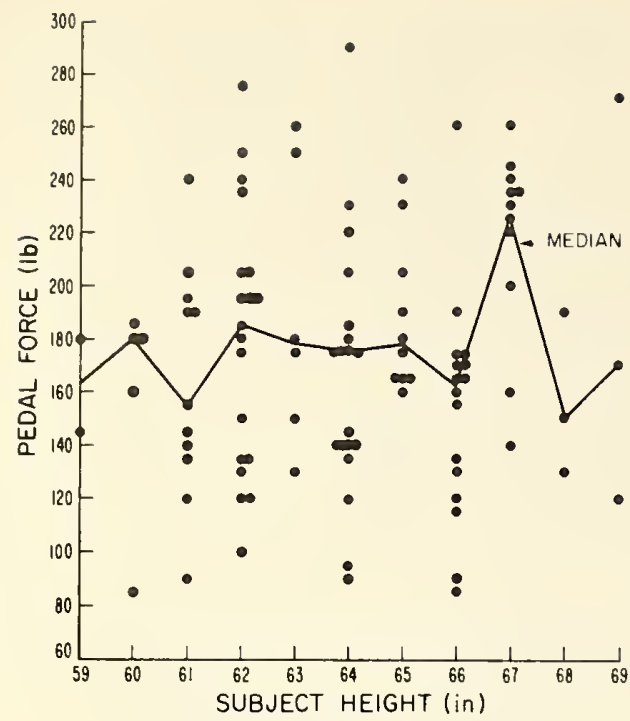

PEDAL FORCE vS SUBJECT HEIGHT FOR APPLICATION 2

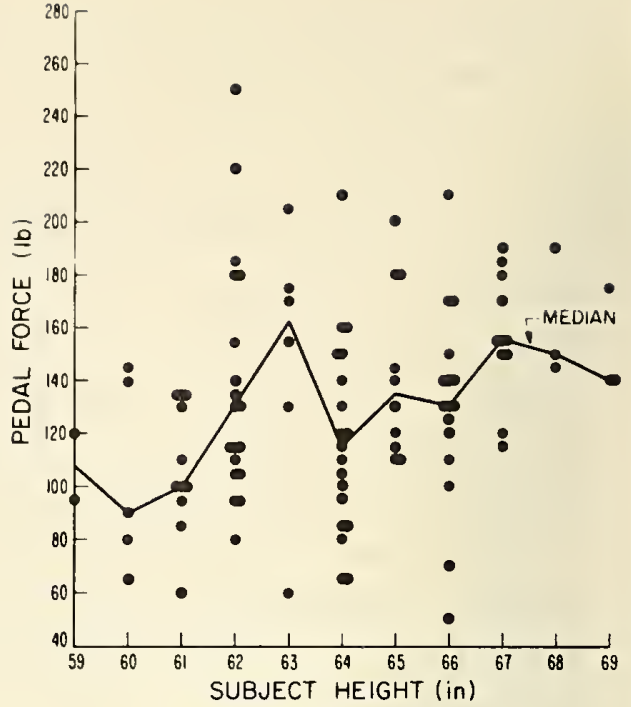

PEDAL FORCE VS SUBJECT HEIGHT FOR APPLICATION 4

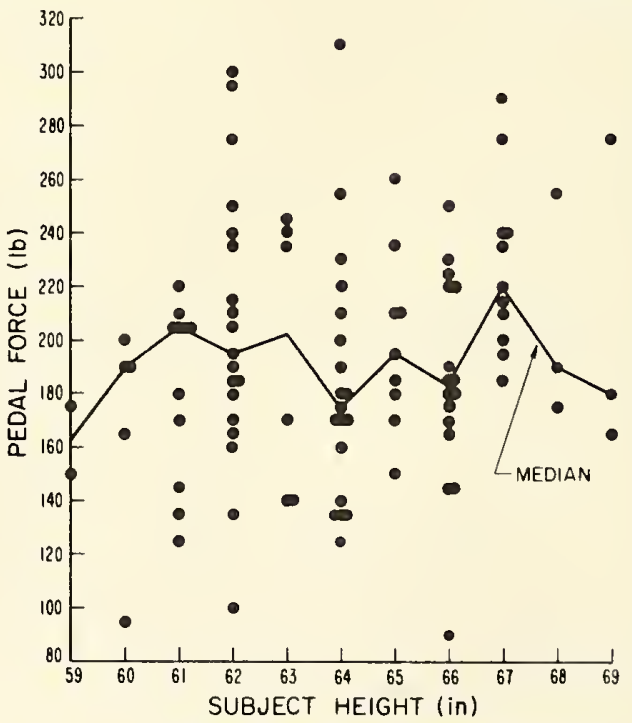

PEDAL FORCE vs SUBJECT HEIGHT

FOR APPLICATION 6 
Serious consideration should be given to reducing the $2001 \mathrm{~b}$ pedal force allowed under certain conditions. Twenty percent of the test subjects could not exceed an average sustained pedal force of $135 \mathrm{lb}$ in vehicle $A$ with a normal pedal height, $100 \mathrm{lb}$ in vehicle $\mathrm{A}$ with a reduced pedal height (simulated failure) or $165 \mathrm{lb}$ in vehicle $\mathrm{B}$ with a normal pedal height. Fifty percent of the test subjects could not achieve an average sustained pedal force of $200 \mathrm{lb}$ in either vehicle.

\section{REFERENCES}

[1] Federal Motor Vehicle Safety Standard No. 105, Hydraulic Service Brake, Emergency Brake, and Parking Brake Systems, Passenger Cars, issued by the U. S. Department of Transportation, Federal Highway Administration, National Highway Safety Bureau, January 1, 1968.

[2] SAE Recommended Practice J937a, Service Brake System Performance Requirements - Passenger Car, 1970 SAE Handbook, Society of Automotive Engineers, 2 Pennsylvania Avenue, New York, New York 10001.

[3] Weight, Height and Selected Body Dimensions of Adults, United States 1960-1962. National Center for Health Statistics, Series 11, Number 8, June 1965. U. S. Department of Health, Education and Welfare. 

Latest developments in the subject area of this publication, as well as in other areas where the National Bureau of Standards is active, are reported in the NBS Technical News Bulletin. See following page. 


\section{HOW TO KEEP ABREAST OF NBS ACTIVITIES}

Your purchase of this publication indicates an interest in the research, development, technology, or service activities of the National Bureau of Standards.

The best source of current awareness in your specific area, as well as in other NBS programs of possible interest, is the TECHNICAL NEWS BULLETIN, a monthly magazine designed for engineers, chemists, physicists, research and product development managers, librarians, and company executives.

If you do not now receive the TECHNICAL NEWS BULLETIN and would like to subscribe, and/or to review some recent issues, please fill out and return the form below.

Mail to: Office of Technical Information and Publications National Bureau of Standards

Washington, D. C. 20234

Name

Affiliation

Address

City State Zip

Please send complimentary past issues of the Technical News Bulletin.

Please enter my 1-yr subscription. Enclosed is my check or money order for $\$ 3.00$ (additional $\$ 1.00$ for foreign mailing). check is made payable to: SUPERINTENDENT OF DOCUMENTS. TN 557 


\section{PERIODICALS}

JOURNAL OF RESEARCH reports National Bureau of Standards research and development in physics, mathematics, chemistry, and engineering. Comprehensive scientific papers give complete details of the work, including laboratory data, experimental procedures, and theoretical and mathematical analyses. Illustrated with photographs, drawings, and charts.

Published in three sections, available separately:

\section{- Physics and Chemistry}

Papers of interest primarily to scientists working in these fields. This section covers a broad range of physical and chemical research, with major emphasis on standards of physical measurement, fundamental constants, and properties of matter. Issued six times a year. Annual subscription: Domestic, $\$ 9.50$; foreign, $\$ 11.73^{*}$.

\section{- Mathematical Sciences}

Studies and compilations designed mainly for the mathematician and theoretical physicist. Topics in mathematical statistics, theory of experiment design, numerical analysis, theoretical physics and chemistry, logical design and programming of computers and computer systems. Short numerical tables. Issued quarterly. Annual subscription: Domestic, $\$ 5.00$; foreign, $\$ 6.25^{*}$.

\section{- Engineering and Instrumentation}

Reporting results of interest chiefly to the engineer and the applied scientist. This section includes many of the new developments in instrumentation resulting from the Bureau's work in physical measurement, data processing, and development of test methods. It will also cover some of the work in acoustics, applied mechanics, building research, and cryogenic engineering. Issued quarterly. Annual subscription: Domestic, $\$ 5.00$; foreign, $\$ 6.25 *$.

\section{TECHNICAL NEWS BULLETIN}

The best single source of information concerning the Bureau's research, developmental, cooperative and publication activities, this monthly publication is designed for the industry-oriented individual whose daily work involves intimate contact with science and technology-for engineers, chemists, physicists, research managers, product-development managers, and company executives. Annual subscription: Domestic, $\$ 3.00$; foreign, $\$ 4.00^{*}$.
NONPERIODICALS

Applied Mathematics Series. Mathematical tables, manuals, and studies.

Building Science Series. Research results, test methods, and performance criteria of building materials, components, systems, and structures.

Handbooks. Recommended codes of engineering and industrial practice (including safety codes) developed in cooperation with interested industries, professional organizations, and regulatory bodies.

Special Publications. Proceedings of NBS conferences, bibliographies, annual reports, wall charts, pamphlets, etc.

Monographs. Major contributions to the technical literature on various subjects related to the Bureau's scientific and technical activities.

National Standard Reference Data Series. NSRDS provides quantitative data on the physical and chemical properties of materials, compiled from the world's literature and critically evaluated.

Product Standards. Provide requirements for sizes, types, quality and methods for testing various industrial products. These standards are developed cooperatively with interested Government and industry groups and provide the basis for common understanding of product characteristics for both buyers and sellers. Their use is voluntary.

Technical Notes. This series consists of communications and reports (covering both other agency and NBS-sponsored work) of limited or transitory interest.

Federal Information Processing Standards Pub-

lications. This series is the official publication within the Federal Government for information on standards adopted and promulgated under the Public Law 89-306, and Bureau of the Budget Circular A-86 entitled, Standardization of Data Elements and Codes in Data Systems.

- Difference in price is due to extra cost of foreign mailing.

Order NBS publications from:

Superintendent of Documents

Government Printing Office

Washington, D.C. 20402 
U.S. DEPARTMENT OF COMMERCE

WASHINGTON, D.C. 20230

OFFICIAL BUSINESS

POSTAGE AND FEES PAID

U.S. DEPARTMENT OF COMMERCE 